\title{
Estimated Effects of the National Breast and Cervical Cancer Early Detection Program on Breast Cancer Mortality
}

\author{
Thomas J. Hoerger, PhD, Donatus U. Ekwueme, PhD, Jacqueline W. Miller, MD, Vladislav \\ Uzunangelov, MS, Ingrid J. Hall, PhD, MPH, Joel Segel, BA, Janet Royalty, MS, James G. \\ Gardner, MSPH, Judith Lee Smith, PhD, and Chunyu Li, PhD \\ Research Triangle Institute International (Hoerger, Uzunangelov, Segel), Research Triangle Park, \\ North Carolina; and the CDC (Ekwueme, Miller, Hall, Royalty, Gardner, Smith, Li), Atlanta, \\ Georgia
}

\begin{abstract}
Background-The National Breast and Cervical Cancer Early Detection Program (NBCCEDP) provides breast cancer screening to medically underserved, low-income women aged 40-64 years. No study has evaluated NBCCEDP's effect on breast cancer mortality.

Purpose-This study estimates life-years saved by NBCCEDP breast cancer screening compared with screening in the absence of NBCCEDP and with no screening.

Methods-A breast cancer simulation model based on existing Cancer Intervention and Surveillance Modeling Network models was constructed. The screening module from these models was modified to reflect screening frequency for NBCCEDP participants. Screening data for uninsured women represented what would have happened without the program. Separate simulations were performed for women who received NBCCEDP (Program) screening, women who potentially received screening without the program (No Program), and women who received no screening (No Screening). The impact of NBCCEDP was estimated as the difference in lifeyears between the Program and No Program, and the Program and No Screening scenarios. The analysis was performed in 2008-2009.
\end{abstract}

Results-Among 1.8 million women who were screened between 1991 and 2006, the Program saved 100,800 life-years compared with No Program and 369,000 life-years compared with No Screening. Per woman screened, the Program saved 0.056 life-years (95\% CI $=0.031,0.081$ ) compared with No Program and 0.206 life-years (95\% CI=0.177, 0.234) compared with No Screening. Per woman with invasive breast cancer and screen-detected invasive cancer, the Program saved 0.41 and 0.71 life-years, respectively, compared with No Program.

Conclusions-These estimates suggest that NBCCEDP breast cancer screening has reduced mortality among medically uninsured and underinsured low-income women.

Address correspondence to: Donatus U. Ekwueme, PhD, CDC, 4770 Buford Highway, MSK-55, Atlanta GA 30341. dce3@ cdc.gov. The findings and conclusions in this report are those of the authors and do not necessarily represent the official position of the CDC. 


\section{Introduction}

Breast cancer is the most commonly diagnosed cancer and the second-leading cause of cancer-related deaths among women in the U.S. ${ }^{1}$ Every year, more than 180,000 women are diagnosed with invasive breast cancer, resulting in more than 40,000 deaths. ${ }^{1}$ Studies ${ }^{2-9}$ have demonstrated that mammography screening can reduce breast cancer mortality rates by 15-30\%. Because of mammography's effectiveness in reducing breast cancer mortality, the U.S. Preventive Services Task Force (USPSTF), other medical organizations, and advocacy groups recommend breast cancer screening for women aged $\geq 40$ years to receive screening mammography every $1-2$ years. ${ }^{4} 10-12$ Recently, the USPSTF revised their earlier recommendation and recommended against routine screening mammography in women aged $<50$ years. ${ }^{13}$ The Task Force recommended that women in their 40 s discuss with their physicians when to start getting mammograms.

Although mammography screening has demonstrated benefits in reducing breast cancer mortality; there is a concern that this recommendation may alienate certain segments of the population and further roll back some of the gains achieved in screening in the past decades. To date, these benefits have not been equally distributed among all women. Poor, uninsured, and underinsured women receive less screening than other groups. ${ }^{14}$ To reduce disparities in breast cancer mortality, the U.S. Congress passed the Breast and Cervical Cancer Mortality Prevention Act (Public Law 101-354) in 1990. This law gave the CDC the authority to establish and administer the National Breast and Cervical Cancer Early Detection Program (NBCCEDP). ${ }^{15}$ The program provides free or low-cost breast cancer screening to medically underserved women aged 40-64 years with an annual income $\_50 \%$ of the federal poverty level. ${ }^{16}$ In addition to screening services, NBCCEDP provides essential functions to reduce morbidity and mortality (e.g., quality assurance, case management, patient navigation, and connection to treatment) following a cancer diagnosis. Since 1991, NBCCEDP has provided more than 4.4 million breast screening examinations to more than 2.3 million women and has diagnosed more than 42,000 breast cancers. ${ }^{15}$

Though these accomplishments may have saved many lives, the program's impact has not been quantified. Quantifying the benefits of mammography screening provided in NBCCEDP would be useful for allocating prevention resources in the program. The present study's objective is to estimate the life-years saved by NBCCEDP breast cancer screening compared with screening in the absence of NBCCEDP and with no screening.

\section{Methods}

A breast cancer simulation model based on existing Cancer Intervention and Surveillance Modeling Network (CISNET) models ${ }^{17-20}$ was constructed. These models contain separate modules, including natural history, screening history, breast cancer mortality, early detection and treatment effects, and other-cause mortality. The screening module was modified to reflect screening frequency for women in NBCCEDP. A technical appendix (available online at www.ajpm-online.net) describes details of each of these modules and underlying parameters and assumptions. A brief explanation of each module is provided below. The analysis was performed in 2008-2009. 


\section{Natural History}

The natural history module determines whether invasive cancer will develop in a woman's lifetime and, if so, when clinical detection will occur in the absence of screening. The probability of cancer developing and the age at clinical detection are based on CISNET estimates using Surveillance Epidemiology and End Results (SEER) breast cancer data. ${ }^{21}$ These estimates approximate breast cancer incidence, stratified by age and birth cohort, in the absence of screening.

\section{Screening}

The screening history module generates a simulated screening history for each woman. The history generates the age of the first mammogram for each woman (some women never receive mammograms). The distribution of first screening and the probability of no screening depend on a woman's age and birth cohort. Once a woman has had one mammogram, future intervals between mammograms are drawn from distributions that depend on whether the woman is an annual, biennial, or irregular screener. The women are randomly assigned to these categories, with the relative probabilities depending on age.

To determine whether breast cancer screening would lead to earlier detection, a breast cancer history and a screening history were generated for each woman. For women in whom cancer developed, the breast cancer history was overlaid with the screening history. If a screen occurred between the age when the tumor was screen detectable and the age at clinical detection, then the tumor would be detected early.

The modified model used CISNET data on the dissemination of mammograms in the general population. ${ }^{17,22}$ The CISNET base case code was obtained and modified to reflect the experience of NBCCEDP participants. NBCCEDP's surveillance database was used to estimate both the women's age at first mammography and the intervals between mammograms for women who participated in the program. National Health Interview Survey data from 1990-2005 was used to estimate mammography patterns among uninsured women to represent mammography screening in the program's absence.

\section{The Effects of Early Detection and Treatment on Breast Cancer Mortality}

The goal of screening is to detect tumors earlier and subsequently provide appropriate and timely treatment. In the model, both early detection and treatment were incorporated in order to evaluate the impact of NBCCEDP. Mortality depends on age, stage, and tumor volume at the time the tumor is discovered. Thus, early detection with screening puts a woman on a more favorable survival curve by lowering tumor volume at discovery and potentially leading to an earlier stage at diagnosis. Survival is also affected by the type of treatment received (including adjuvant multiagent chemotherapy and tamoxifen), which depends on tumor stage at detection and estrogen receptor (ER) status. A woman is assumed to die from breast cancer if her age at detection plus her survival time is earlier than her estimated date of death from other causes. 


\section{Mortality from Other Causes}

Each woman is randomly assigned a date of death from other causes based on general U.S. mortality rates for 1950-2004. Breast cancer mortality is subtracted from the general mortality rates using methods developed by CISNET. ${ }^{23} \mathrm{~A}$ woman with breast cancer is assumed to die from other causes if her estimated date of death from other causes is earlier than her age at cancer detection plus her estimated survival time.

\section{Calculation of Life-Years Saved}

The analysis was limited to women who received mammograms covered by NBCCEDP between 1997 and 2006 because the NBCCEDP database was not complete prior to 1997. Using the screening history parameters, life-years saved by the program during the entire period from 1991 to 2006 and for their remaining lifetime were estimated.

Separate Monte Carlo simulations were performed for women who received screening through NBCCEDP (Program), women who potentially received screening without the program (No Program), and women who received no screening at all (No Screening). To estimate mean values and CIs for life-years saved, 1000 samples with 100,000 women per sample were simulated. To compare simulation results with real-world outcomes, simulation results were stated in terms of a population of 1.8 million women (i.e., the number of women who received mammograms through NBCCEDP between 1997 and 2006). The effect of NBCCEDP was estimated as the difference in life-years between the Program and No Program. This comparison reflects the fact that some uninsured women may receive mammogram screening even in the absence of NBCCEDP. To estimate the overall impact of screening, the difference in life-years between the Program and No Screening were calculated. A bootstrap approach was used to calculate $95 \%$ CIs for life-years saved, taking the 25th and 975th values from the 1000 samples. The model was programmed in TreeAge Pro 2008, version 1.5.1 (TreeAge Software Inc, Williamstown MA).

\section{Sensitivity Analysis}

Univariate sensitivity analyses were conducted from 2008 to 2009 to assess the impact of alternative assumptions about frequency of screening through the Program, the median diameter for screen detection, trends in breast cancer incidence, treatment efficacy, and the potential benefits from earlier clinical detection of cancers with symptoms through the Program. Each sensitivity analysis was based on 30 samples of 60,000 women.

\section{Results}

Based on the three simulation runs, women participating in NBCCEDP will receive 17.17 screening mammograms over their lifetimes, compared with 12.38 screening mammograms in No Program scenario, and by definition, 0 screening mammograms in No Screening scenario (Table 1). Women who were eligible for the Program (aged 40-64 years) during at least some years of the period from 1991 to 2006 were estimated to receive 4.26 screenings with the Program compared with 2.44 screenings with No Program. Many women were eligible only during some of the period (e.g., a woman born in 1961 would be eligible from 2001 to 2006 only). On average, actual NBCCEDP participants received 1.95 screenings 
through the program, slightly higher than the estimated 1.82 screenings difference between the Program and No Program. The Program estimates include mammograms paid for by the program and mammograms received outside the program, so the difference between the Program and No Program scenario estimates represents the program's incremental effect.

Slightly more invasive cancers are detected through screening or clinically with the Program than would be detected under the No Program and No Screening scenarios. The difference between the Program and No Screening scenarios represents tumors that are detected through screening that would not have been detected clinically until after the woman had died of other causes (e.g., a woman diagnosed at age 64 years in the Program may die from other causes at age 65 years; her tumor may not be evident prior to death in the No Screening scenario). About 57\% (7.9\%/13.8\%) of the invasive cancers are screen-detected in the Program compared with about $46 \%(6.3 \% / 13.8 \%)$ screen-detected in the No Program scenario. The estimated number of screen-detected cancers for women aged 40-64 years between 1991 and 2006 was 28,851 with the Program compared with 18,596 with No Program. The difference 10,255 approximates the 9037 invasive cancers detected among actual NBCCEDP participants who underwent nonsymptomatic screening during the period (Table 1).

In the Program scenario, 3.7\% of women died of breast cancer compared with $4.0 \%$ and $5.0 \%$ in the No Program, and No Screening scenarios, respectively. The average age at death was $80.57,80.52$, and 80.37 years for the Program, No Program, and No Screening scenarios, respectively (Table 1). The Program saved 100,800 life-years relative to No Program and 370,800 life-years relative to No Screening. Per woman screened, the Program saved 0.056 life-years (or 20.4 days) compared with No Program and 0.206 life-years (or 75.2 days) compared with No Screening. Per woman with invasive breast cancer and screendetected invasive cancer, the Program saved 0.41 and 0.71 life-years, respectively, compared with No Program.

The $95 \%$ CI for life-years saved by the Program relative to No Program ranges from 0.031 to 0.081 years, whereas the interval for the Program relative to No Screening ranges from 0.177 to 0.234 years.

\section{Sensitivity Analyses}

Table 2 shows the results of one-way sensitivity analyses. Because the analyses were run for 30 samples of 60,000 women, rather than the 1000 samples of 100,000 women in Table 1 (due to run-time constraints), the main analysis estimate (0.061) in Table 2 is slightly different from the estimate (0.056) in Table 1 . This difference, which is due to random variation, does not affect the qualitative results of the sensitivity analyses.

The main analysis assumes that substantially more women will be annual and biennial screeners through the Program than through No Program. If the Program advantage in annual and biennial screening is cut in half, the life-years saved by the Program declines to 0.044. The life-years saved by the Program declines to 0.053 if all participating women receive biennial screening. Conversely, if all Program women receive annual screening, life- 
years saved increases to 0.089 . This may be viewed as the greatest potential gain from screening through the Program.

In the main analysis, the median tumor diameter for screening detection was $10 \mathrm{~mm}$. Increasing the median diameter to $15 \mathrm{~mm}$ reduced life-years gained from the Program to 0.048. Conversely, if the median diameter for detection was $5 \mathrm{~mm}$, life-years gained increased to 0.064 . The reduction in tumor detection size will provide benefits only if screening occurs during the interval of time when the tumor's diameter is between 5 and 10 $\mathrm{mm}$. The length of this interval depends on the tumor's growth rate.

The analysis incorporates the increasing incidence of breast cancer between 1980 and 2000 as suggested by CISNET. Slower rates of increase in the incidence of invasive cancer were also applied (or equivalently, larger percentages of ductal carcinoma in situ [DCIS] were subtracted). With lower incidence of invasive cancers, the difference in life-years between the Program and No Program scenarios declined moderately.

Model treatment effects are based on therapies available in 2000, and therapies may have improved over time. Several analyses assessed the effects of potential changes in treatment. When the mortality hazard was reduced by $10 \%, 20 \%$, and $30 \%$ for both clinical and screendetected cancers, the difference in life-years saved between the Program and No Program scenarios fell to $0.057,0.053$, and 0.049 years, respectively.

The main analysis of Program benefits included life-years gained from early detection only. However, the Program may encourage uninsured women to seek care earlier when they experience symptoms of potential breast cancer, such as lumps or prolonged tenderness. This could lead to additional life-years saved if diagnosis and treatment for these clinically detected cancers begins earlier. The potential benefits that would occur if the Program allowed clinical detection to occur 1, 2, 3, or 6 months earlier were calculated. Adding benefits from clinically detected cancers would increase substantially the life-years saved by the Program. If clinical detection occurred 1 month earlier, the Program would save 0.090 life-years, nearly $50 \%$ higher than the savings from screening detection only. Life-years increased even more when clinical detection occurred 2, 3, or 6 months earlier.

\section{Discussion}

This is the first study to estimate the effectiveness of NBCCEDP on breast cancer mortality measured by life-years saved due to screening over the past 15 years. Compared with No Screening, women screened through NBCCEDP gained 0.056 life-years per woman. Because the women diagnosed with breast cancer through NBCCEDP-sponsored screening programs are low-income, uninsured or underinsured, and less able to afford screening, these findings underscore the importance of organized screening programs such as NBCCEDP to the most vulnerable members of the population who are eligible for screening. Improving health for vulnerable members of the population by eliminating health disparities is one of the two overarching goals of the Healthy People 2010's agenda for health promotion and disease prevention in the U.S. ${ }^{24}$ 
At first glance, the estimated 0.056-life-year gain per woman in the Program might appear to be small. However, this population-based average of the 1.8 million women screened through the Program represents larger gains for women who actually develop breast cancer. The estimated Program gain for women in whom breast cancer develops is 0.41 life-years, and the gain for women in whom breast cancer is detected early through screening is 0.71 life-years. The population average appears small because (1) breast cancer does not develop in seven of eight women; (2) screening detects only about $57 \%$ of cancers early; (3) some women in whom tumors are screen-detected early would have survived in the absence of screening; and (4) some women in whom tumors are screen-detected early still die.

Averaging across all women also masks the large gains for women in whom cancers were screen-detected and who would have died of breast cancer in the absence of the Program. In a simulation of 100,000 women, 979 fell into this category, with an average gain of 11.33 life-years.

Comparing results described in this report with other national prevention programs is difficult because study methods, target population ages, and disease characteristics differ. Regardless of these differences, Table 3 shows expected improvements in life-years from selected population-based prevention programs ${ }^{25}$ and the estimates for life-years saved by NBCCEDP. The table provides some perspective as well as consistency for the results. On a per-person basis, an earlier study ${ }^{25}$ reported gain in life-years for 10 years of biennial mammography screening starting at age 50 years to be 0.067 , which is consistent with the present study's results. Breast cancer screening through NBCCEDP gains fewer life-years than stopping smoking (0.056 vs $0.67-0.83$ life-year per person) but more life-years than childhood immunization vaccines, such as measles and rubella (0.008 life-year each). Further, a recent regression-based modeling study examined the impact of NBCCEDP on breast cancer mortality rates and found that there is some evidence to suggest that NBCCEDP has led to a reduction in breast cancer mortality rates, which is also consistent with the present study's results. ${ }^{26}$

The analysis has some limitations. First, the results are based on a simulation model rather than clinical trial results or a long-term, follow-up study of health outcomes for program participants. A clinical trial of NBCCEDP is unlikely for ethical reasons. Currently, longterm, follow-up data are not collected on women who receive NBCCEDP screening. Second, the analysis assumes that NBCCEDP participants in whom cancers are detected receive the same treatment as women in the general population. Although complete treatment data on NBCCEDP participants are not collected, program data confirm that most women with a diagnosis of breast cancer begin definitive treatment. Since 2000, nearly all women diagnosed through the program have been eligible for immediate enrollment in Medicaid, wherein complete treatment is provided free.

Third, the results show only life-years gained and do not include gains from reductions in morbidity, which would increase the quality-adjusted life-years (QALYs) gained by NBCCEDP. For most cancers, however, QALYs gained are largely determined by life-years gained. ${ }^{27}$ On the other hand, the analysis does not include harm arising from false positives or overdiagnosis (the identification of tumors that would never have become a clinical problem in the patient's lifetime), which might reduce the QALYs gained. Fourth, the main 
analysis includes only life-years saved by screening mammograms. As the sensitivity analyses show, life-years saved would be higher if access to the Program led to earlier clinical diagnosis for women who seek screening in response to breast cancer symptoms. However, data to accurately simulate symptomatic screening were lacking.

Fifth, the NBCCEDP's database is incomplete before 1997. Therefore, the analysis focused on women who received at least one NBCCEDP mammogram between 1997 and 2006. Available earlier data on these women were used to estimate the benefits they received for the entire period between 1991 and 2006. The estimates may underestimate the total lifeyears saved by the Program because the approach excluded women who received Program screenings between 1991 and 1996 only. Sixth, NBCCEDP may help uninsured women gain access to the healthcare system, leading to better care for conditions besides breast and cervical cancer. For instance, the Well-Integrated Screening and Evaluation for Women Across the Nation (WISEWOMAN) ${ }^{28}$ program provides heart disease and stroke screening to women who enter NBCCEDP. The analysis includes only the direct effects of NBCCEDP on breast cancer survival. Finally, the analysis focuses on the effects of NBCCEDP screening. Breast cancer outcomes depend on other public health initiatives, including programs to reduce obesity, lower smoking rates, and increase awareness of breast cancer and screening guidelines. These initiatives are not explicitly modeled in the analysis.

These limitations notwithstanding, the estimates of life-years saved suggest that the collective efforts of NBCCEDP grantees, policymakers, and other national partners to offer breast cancer screening to medically underserved, low-income populations have had substantial impact in reducing mortality from breast cancer for these populations. For researchers, these results can be used to estimate the cost effectiveness of breast cancer screening in this population. For policymakers, the findings in this article are encouraging and may be useful for allocating resources in the program. Currently, less than $15 \%$ of women eligible for NBCCEDP receive mammography screening from the program. ${ }^{29}$ If the number of women served by the program increases, life-years saved will increase proportionately. Alternatively, medically underserved low-income women may gain better access to life-saving cancer screening services through implementation of the Affordable Care Act.

\section{Supplementary Material}

Refer to Web version on PubMed Central for supplementary material.

\section{Acknowledgments}

This research was supported by funding (Contract \#200-2002-00575) from the Centers for Disease Control and Prevention (CDC).

\section{References}

1. U.S. Cancer Statistics Working Group. US Cancer Statistics: 2005 Incidence and Mortality. Atlanta GA: USDHHS, CDC and National Cancer Institute; 2010. www.cdc.gov/uscs 
2. Mandelblatt JS, Cronin KA, Bailey S, et al. Effects of mammography screening under different screening schedules: model estimates of potential benefits and harms. Ann Intern Med. 2009; 151:738-47. [PubMed: 19920274]

3. Humphrey LL, Helfand M, Chan BK, Woolf SH. Breast cancer screening: a summary of the evidence for the U.S. Preventive Services Task Force. Ann Intern Med. 2002; 137:347-60. [PubMed: 12204020]

4. U S. Preventive Services Task Force (USPSTF). Screening for breast cancer: recommendations and rationale. Ann Intern Med. 2002; 137:344-46. [PubMed: 12204019]

5. Berry DA, Cronin KA, Plevritis SK, et al. Effects of screening and adjuvant therapy on mortality from breast cancer. N Engl J Med. 2005; 353:1784-92. [PubMed: 16251534]

6. Mandelblatt JS, Yabroff KR. Effectiveness of interventions designed to increase mammography use: a meta-analysis of provider-targeted strategies. Cancer Epidemiol Biomarkers Prev. 1999; 8:759-67. [PubMed: 10498394]

7. Fletcher SW, Elmore JG. Mammographic screening for breast cancer. N Engl J Med. 2003; 348:1672-80. [PubMed: 12711743]

8. Nyström L, Andersson I, Bjurstam N, Frisell J, Nordenskjold B, Rutqvist LE. Long-term effects of mammography screening: updated overview of the Swedish randomised trials [published erratum appears in Lancet 2002;360(9334):724]. Lancet. 2002; 359(9310):909-19. [PubMed: 11918907]

9. Elwood JM, Cox B, Richardson AK. The effectiveness of breast cancer screening by mammography in younger women. Online J Curr Clin Trials. 1993 Feb 25. Doc No. 32: 23,227 words.

10. Smith RA, Saslow D, Sawyer KA, et al. American Cancer Society guidelines for breast cancer screening: update 2003. CA Cancer J Clin. 2003; 54:141-69.

11. Feig SA, Kopans DB, Sickles EA, Jackson VP, Monsees B. Rationale for annual screening mammography for women ages 40-49 years. Breast Dis. 1998; 10(3-4):13-21. [PubMed: 15687560]

12. Nelso HD, Tyne K, Naik A, Bougatsos C, Chan BK, Humphrey L. Screening for breast cancer: an update for the U.S. Preventive Services Task Force. Ann Intern Med. 2009; 151:727-37. [PubMed: 19920273]

13. U S. Preventive Services Task Force. Screening for breast cancer: U.S Preventive Services Task Force recommendation statement. Ann Intern Med. 2009; 151:716-26. [PubMed: 19920272]

14. Edwards BK, Ward E, Kohler BA, et al. Annual report to the nation on the status of cancer, 19752006, featuring colorectal cancer trends and impact of interventions (risk factors, screening, and treatment) to reduce future rates. Cancer. 2010; 116:544-73. [PubMed: 19998273]

15. CDC. National Breast and Cervical Cancer Early Detection Program. www.cdc.gov/cancer/ nbccedp/about.htm

16. Eheman CR, Benard VB, Blackman D, et al. Breast cancer screening among low-income or uninsured women: results from the National Breast and Cervical Cancer Early Detection Program, July 1995 to March 2002 (U.S). Cancer Causes Control. 2006; 17:29-38. [PubMed: 16411050]

17. Cronin KA, Yu B, Krapcho M, et al. Modeling the dissemination of mammography in the U.S. Cancer Causes Control. 2005; (16):701-12. [PubMed: 16049809]

18. Plevritis SK, Salzman P, Sigal BM, Glynn P. A natural history model of stage progression applied to breast cancer. Stat Med. 2007; (26):581-95. [PubMed: 16598706]

19. Plevritis SK, Sigal BM, Salzman P, Rosenberg J, Glynn P. A stochastic simulation model of U.S. breast cancer mortality trends from 1975 to 2000. J Natl Cancer Inst Monogr. 2006; Chapter 12(36):86-95.

20. Feuer EJ. Modeling the impact of adjuvant therapy and screening mammography on U.S. breast cancer mortality between 1975 and 2000: introduction to the problem. J Natl Cancer Inst Monogr. 2006; (36):2-6. [PubMed: 17032887]

21. Holford TR, Cronin KA, Mariotto AB, Feuer EJ. Changing patterns in breast cancer incidence trends. J Natl Cancer Inst Monogr. 2006; Chapter 4(36):19-25.

22. Cronin KA, Mariotto AB, Clarke LD, Feuer EJ. Additional common inputs for analyzing impact of adjuvant therapy and mammography on U.S. mortality. J Natl Cancer Inst Monogr. 2006; Chapter $5(36): 26-9$. 
23. Rosenberg M. Competing risks to breast cancer mortality. J Natl Cancer Inst Monogr. 2006; Chapter 3(36):15-9.

24. USDHHS. Healthy People 2010: understanding and improving health. 2. Washington DC: U.S. Government Printing Office; 2003.

25. Wright JC, Weinstein MC. Gains in life expectancy from medical interventions-standardizing data on outcomes. N Engl J Med. 1998; 339:380-6. [PubMed: 9691106]

26. Howard DH, Ekwueme DU, Gardner JG, Tangka FL, Li C, Miller JW. The impact of a national program to provide free mammograms to low-income, uninsured women on breast cancer mortality rates. Cancer. 2010; 116(19):4456-62. [PubMed: 20564744]

27. Tengs TO. Cost-effectiveness versus cost-utility analysis of interventions for cancer: does adjusting for health-related quality of life really matter? Value Health. 2004; 7(1):70-8. [PubMed: 14720132]

28. CDC. WISEWOMAN: Well-Integrated Screening and Evaluation for Women Across the Nation. www.cdc.gov/WISEWOMAN/

29. Tangka FKL, Dalaker J, Chattopadhyay SK, et al. Meeting the mammography screening needs of underserved women: the performance of the National Breast and Cervical Cancer Early Detection Program in 2002-2003 (U.S). Cancer Causes Control. 2006; 17:1145-54. [PubMed: 17006720]

\section{Appendix. Supplementary data}

Supplementary data associated with this article can be found, in the online version, at doi: 10.1016/j.amepre.2010.12.017. 


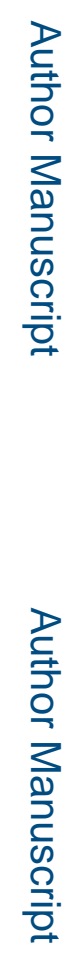

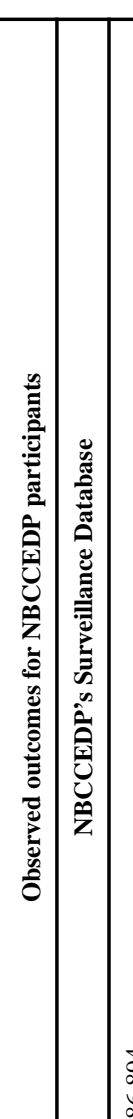

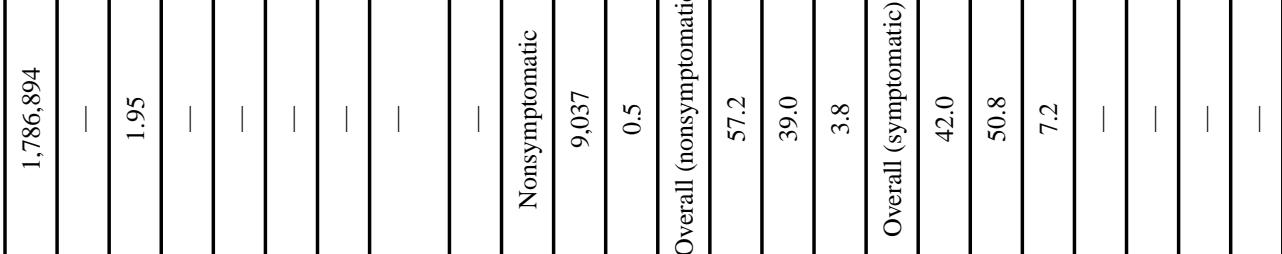

?

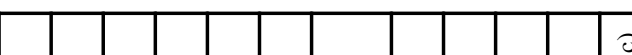

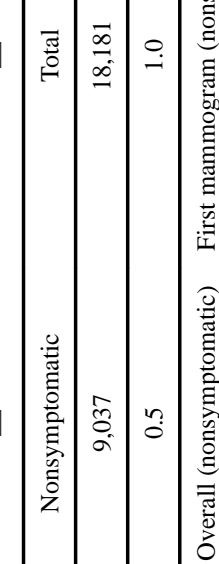

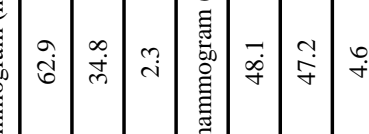

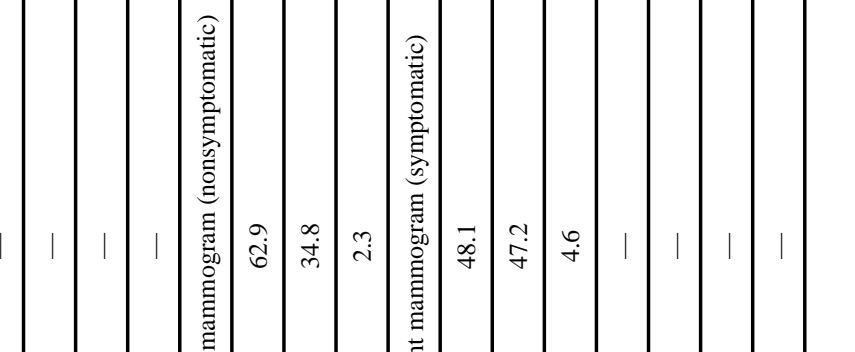

窎

(2)

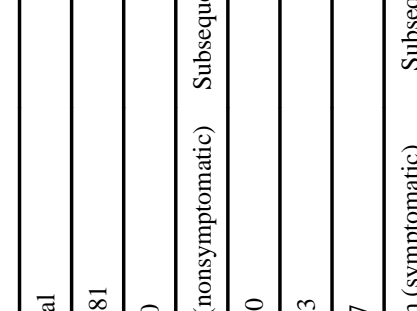

䐻

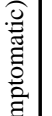

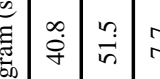

畜

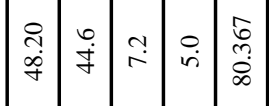
$\frac{2}{5}$

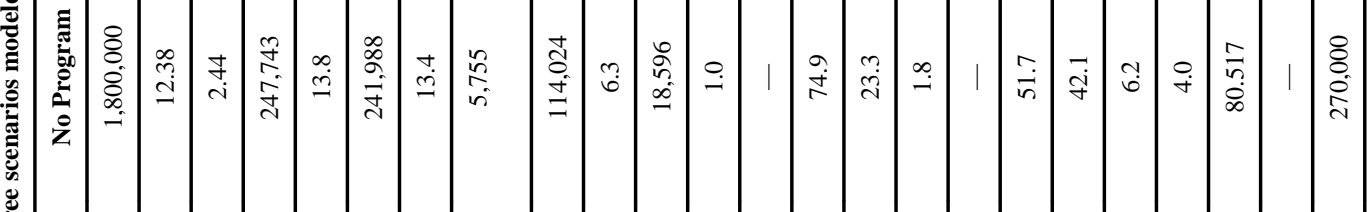
$\bar{F}$

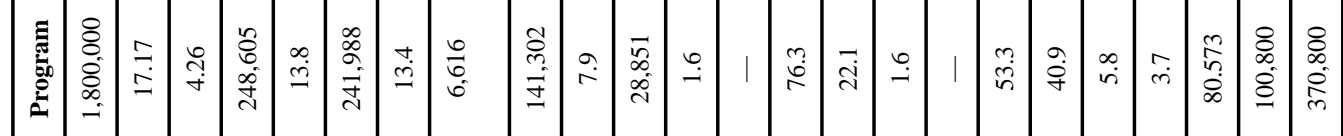

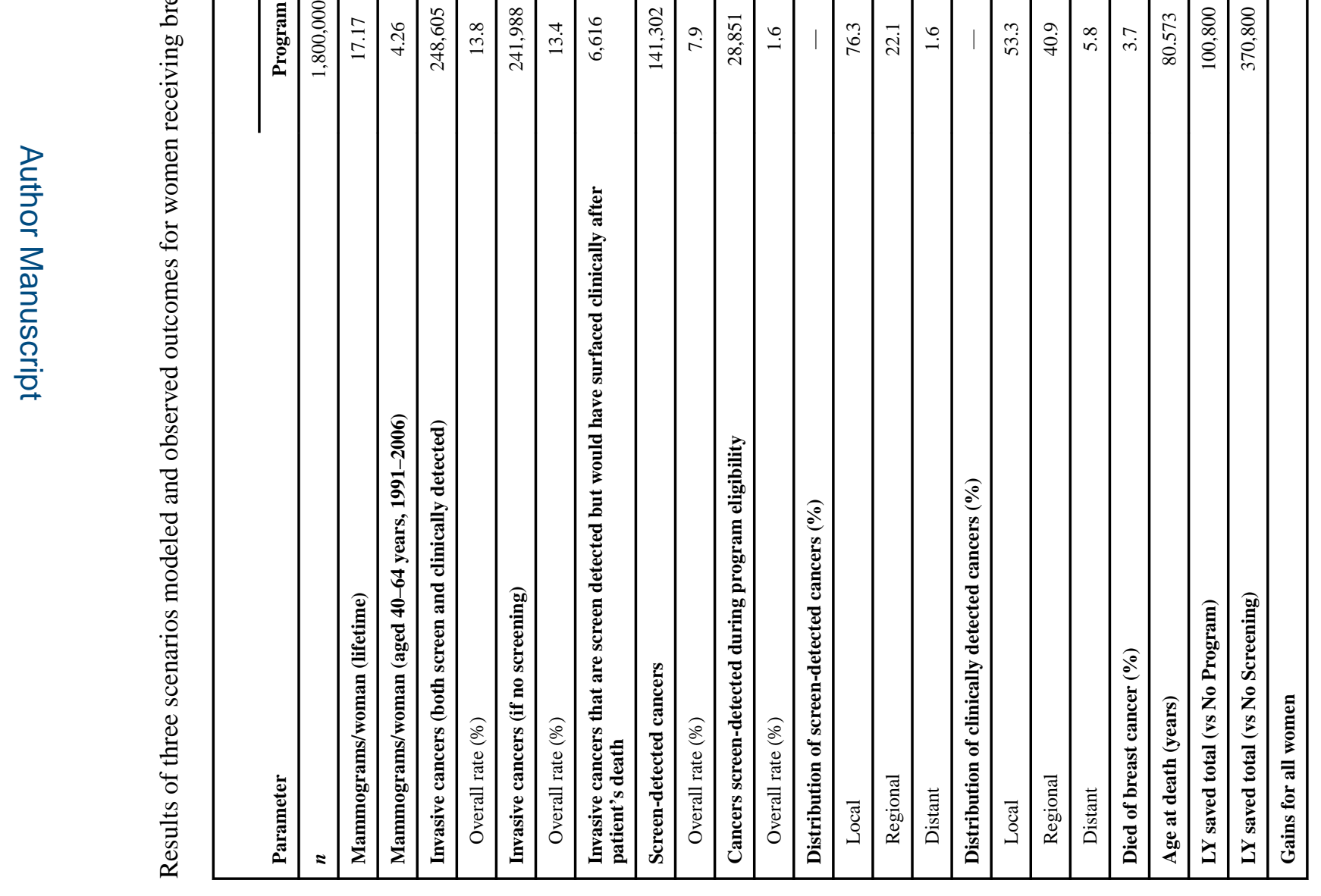




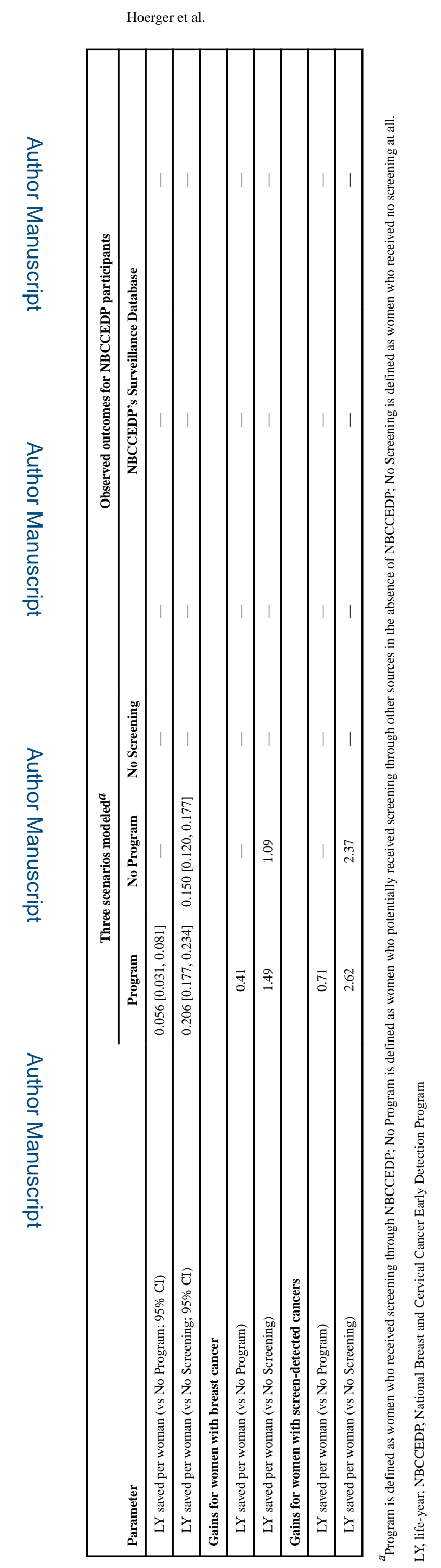




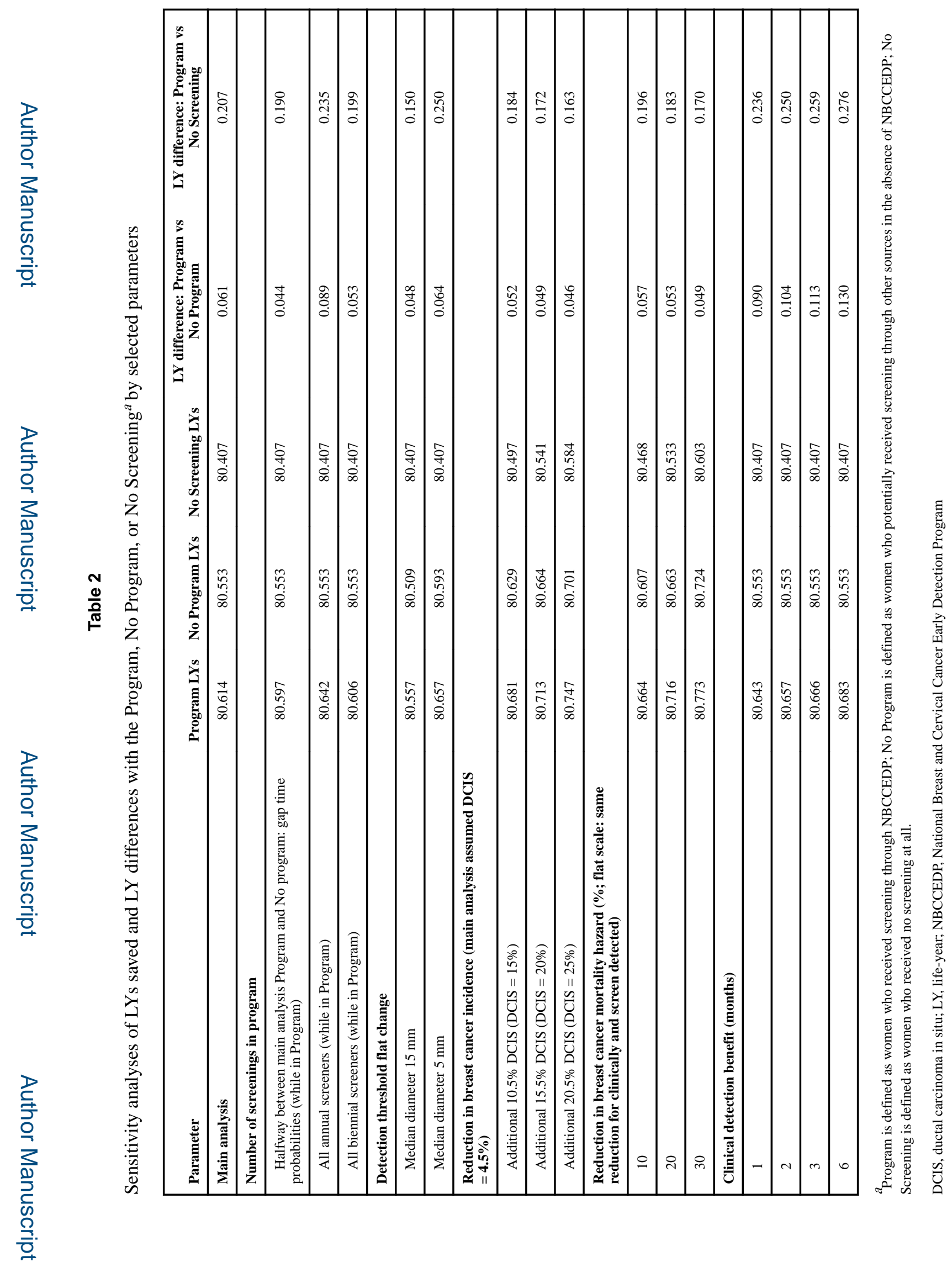

Am J Prev Med. Author manuscript; available in PMC 2018 March 09. 
Table 3

Life years saved by selected preventive services

\begin{tabular}{|llc|}
\hline Intervention & Target population & $\begin{array}{c}\text { Gain in life expectancy (life- } \\
\text { years) }\end{array}$ \\
\hline Quitting cigarette smoking & 35 -year-olds & $0.667-0.833$ \\
\hline Pap, every year for 55 years & 20-year-old women & 0.267 \\
\hline Annual fecal occult blood test, plus barium enema or colonoscopy & 50-year-olds & $0.183-0.208$ \\
\hline 10 years of biennial mammography & 50-year-old women & 0.067 \\
\hline NBCCEDP (Program vs No Program) & Low-income, uninsured women, aged & $\mathbf{0 . 0 5 6}$ \\
\hline Measles vaccine & Inf-64 years & 0.008 \\
\hline Rubella vaccine & Infants & 0.008 \\
\hline
\end{tabular}

Source: NBCCEDP: present study. All other interventions: Wright and Weinstein, $1998 .{ }^{25}$

NBCCEDP, National Breast and Cervical Cancer Early Detection Program 